\title{
BOUNDED GEODESICS OF RIEMANN SURFACES AND HYPERBOLIC MANIFOLDS
}

\author{
J. L. FERNÁNDEZ AND M. V. MELIÁN
}

\begin{abstract}
We study the set of bounded geodesics of hyperbolic manifolds. For general Riemann surfaces and for hyperbolic manifolds with some finiteness assumption on their geometry we determine its Hausdorff dimension. Some applications to diophantine approximation are included.
\end{abstract}

\section{INTRODUCTION}

0.1. A Riemann surface $\mathscr{R}$, other than the sphere, the plane, the cylinder and the tori, has the upper half plane $\mathbb{H}^{2}=\{z=x+\sqrt{-1} y \in \mathbb{C}: y>0\}$ as its universal cover, and thus can be given as a quotient of $\mathbb{H}^{2}$ by a Fuchsian group $\Gamma$, i.e., a discrete group of Möbius transformations of $\mathbb{H}^{2}$. The group $\operatorname{Möb}\left(\mathbb{H}^{2}\right)$ of Möbius transformations of $\mathbb{H}^{2}$ is also the group of (orientation preserving) isometries of the Poincaré metric of $\mathbb{H}^{2}: d s^{2}=y^{-2}\left(d x^{2}+d y^{2}\right)$, and thus one may projects this metric onto $\mathscr{R}$, and $\mathscr{R}$ becomes a complete Riemannian surface of constant negative curvature. Conversely, such a surface can be endowed with a conformal structure in a canonical way by means of isothermal coordinates. It is well known that these correspondences of structures are inverses of each other.

In this paper the term Riemann surface means a complete Riemannian surface of constant negative curvature or a Riemann surface with the upper half plane as universal cover. We shall frequently switch between the upper half plane $\mathbb{H}^{2}$ and the unit disk $\mathbb{D}$ models of hyperbolic geometry.

Given a point $p$ on a Riemann surface $\mathscr{R}$, we denote by $S(p)$ the unit circle of the tangent plane of $\mathscr{R}$ at $p$, and for every $v \in S(p)$ we let $\gamma_{v}(t)$ denote the unit speed geodesic emanating from $p$ at time 0 in the direction $v$. If such a geodesic ray remains at a bounded distance from the starting point $p$ at all times $t \geq 0$ we call it a bounded geodesic. We denote by $\mathscr{B}(\mathscr{R}, p)$ or $\mathscr{B}(p)$ the set of directions $v \in S(p)$ such that the geodesic $\gamma_{v}(t)$ is bounded.

The aim of this paper is to prove the following

Theorem 1. For any Riemann surface $\mathscr{R}=\mathbb{D} / \Gamma$, other than the punctured disk, and every $p \in \mathscr{R}$ the Hausdorff dimension of $\mathscr{B}(\mathscr{R}, p)$ is $\delta(\Gamma)$. If $\mathscr{R}$ is the punctured disk then $\mathscr{B}(\mathscr{R}, p)$ is empty.

Received by the editors July 13, 1994 and, in revised form, October 7, 1994.

1991 Mathematics Subject Classification. Primary 30F35, 30F40.

Research of the first author supported by a grant from DGICYT, Ministerio de Educación y Ciencia, Spain, and the Human Capital and Mobility of the European Union.

Research of the second author supported by Beca de Formación de Personal Investigador, Ministerio de Educación y Ciencia, Spain, Universidad Autónoma de Madrid. 
Hereafter $H D$ will denote Hausdorff dimension with respect to the natural induced distance in $S(p)$. As usual we shall denote by $\delta(\Gamma)$ or $\delta(\mathscr{R})$ the exponent of convergence of the Fuchsian group $\Gamma$, or the Riemann surface $\mathscr{R}$, which is defined as

$$
\delta(\Gamma)=\inf \left\{\alpha>0: \sum_{\gamma \in \Gamma}(1-|\gamma(0)|)^{\alpha}<+\infty\right\} .
$$

We refer to [C] or [R] for definitions and background on Hausdorff dimension and $\alpha$-dimensional content. For background concerning Möbius groups we refer to $[\mathrm{A}],[\mathrm{B}]$ and $[\mathrm{N}]$.

Remark. By the way, the case of exceptional Riemann surfaces endowed with their canonical geometries is very simple. $\widehat{\mathbb{C}}$ and the tori are compact surfaces. In the plane no geodesic is bounded, while in the cylinder only two geodesics from each point are bounded.

These results originate in a classical theorem of Jarník [J]. This theorem states that the Hausdorff dimension of the set of real numbers which are badly approximable by rational numbers, i.e., those $x \in \mathbb{R}$ such that

$$
\left|x-\frac{p}{q}\right| \geq \frac{c(x)}{q^{2}}
$$

for every pair $p, q$ of relative prime integers, (e.g., the golden ratio), has Hausdorff dimension one. Jarnik's theorem can be interpreted in terms of the set of bounded geodesics emanating from a fixed point of the surface $\mathscr{M}=$ $\mathbb{H}^{2} / S L(2, \mathbb{Z})$. Here $S L(2, \mathbb{Z})$ is the group of $2 \times 2$ matrices with integer entries and on the quotient $\mathscr{M}$ one projects the Poincare metric of $\mathbb{H}^{2}$.

Riemann surfaces $\mathscr{R}$ of finite area have exponent of convergence $\delta(\mathscr{R})=1$ and it was known that the set of directions $v \in S(p)$ such that $\gamma_{v}$ is bounded has Hausdorff dimension one. This is a consequence of Patterson's extension [P1] of Jarnik's theorem. This result was further extended by Dani [D] who showed that for a complete Riemannian manifold of constant negative sectional curvature and finite volume the set of directions $v \in S(p)$ such that $\gamma_{v}$ is bounded has full Hausdorff dimension. Some particular cases are considered in [M]. Our Theorem 1 completes the picture in the two-dimensional case.

Similarly, following the proof of Theorem 1 one also obtains analogous results in higher dimensions. For $n=3$ one has the following

Theorem 1'. For any geometrically finite hyperbolic 3-manifold $\mathscr{M}=\mathbb{H}^{3} / \Gamma$, except the quotients of purely parabolic groups, and every $p \in \mathscr{M}$ the Hausdorff dimension of $\mathscr{B}(\mathscr{M}, p)$ is $\delta(\Gamma)$.

This result also holds for $\mathscr{M}=\mathbb{H}^{3} / \Gamma$ with $\Gamma$ an increasing union of a sequence of geometrically finite Kleinian groups.

For general dimension, a proof similar to Theorem 1 gives the same conclusion under the assumption that not all cusps have non-maximal rank, which in particular, includes the theorem of Dani quoted above.

Once this work was completed and the manuscript ready, we received from B. Stratmann a preprint of his where he also studies the Hausdorff dimension of 
the set of bounded geodesics of finite type hyperbolic manifolds. Our result is stronger in dimension 2 since it is valid for an arbitrary Riemann surface with no finiteness assumption. On the other hand, for dimensions greater than 4 we require an extra assumption concerning the rank of the cusps of the manifolds. The proofs are different. Also, more recently, C. Bishop and P. Jones have been able to show in [BJ] that Theorem $1^{\prime}$ is true in any dimension and with no finiteness assumption.

Our strategy for the proof of Theorem 1 is the following: In section 1 we will show how to reduce the proof to the case of Riemann surfaces of finite type, i.e., with finitely generated fundamental group. That case is further divided into two cases. If there are no cuspidal ends the proof is quite easy and it is described in section 2. In the more interesting case of Riemann surfaces with cusps we shall obtain a more precise result (Theorem 2 ), which is the content of section 3. The higher dimensional version, Theorem $1^{\prime}$, is explained in section 5.

0.2. Connection with the conical limit set. Write $\mathscr{R}=\mathbb{D} / \Gamma$, where $\Gamma$ is a Fuchsian group acting on the unit disk $\mathbb{D}$ with no elliptic elements. Recall the definitions of the limit set $\Lambda(\Gamma)$ and the conical limit set $\Lambda_{c}(\Gamma)$ and also the well-known fact that the Hausdorff dimension of the conical limit set of a Fuchsian group $\Gamma$ coincides with the exponent $\delta(\Gamma)$, see, e.g., [N]. Now, we introduce a subset, denoted $\Lambda_{b}(\Gamma)$, of the conical limit set as follows, we say that $e^{i \theta} \in \partial \mathbb{D}$ belongs to $\Lambda_{b}(\Gamma)$ if there exist $\rho=\rho\left(e^{i \theta}\right)<1, d=d\left(e^{i \theta}\right)>0$ and a sequence $\left\{\gamma_{n}\right\}$ of elements of $\Gamma$ such that

$$
\text { (i) }\left|e^{i \theta}-\gamma_{n}(0)\right|<c\left(1-\left|\gamma_{n}(0)\right|\right)
$$

and

$$
\text { (ii) } \frac{1}{d} \rho^{n} \leq\left(1-\left|\gamma_{n}(0)\right|\right) \leq d \rho^{n} \text {. }
$$

Notice that the first condition alone determines the conical limit points. It is clear that if we use any other point $z \in \mathbb{D}$ instead of 0 we obtain the same set.

Geometrically, the conditions above simply mean that

$$
\sup _{0 \leq r<1} \operatorname{dist}\left(r e^{i \theta}, \Gamma(0)\right)<\infty
$$

or, equivalently, that the geodesic in $\mathbb{D}$ from 0 (or $z \in \mathbb{D}$ ) ending at $e^{i \theta}$ remains at all times at a bounded distance from the orbit of 0 (or $z$ ). Here dist means hyperbolic distance in $\mathbb{D}$.

Now, if $\gamma_{v}$ is a geodesic on $\mathscr{R}=\mathbb{D} / \Gamma$ and if we let $\tilde{\gamma}_{v}$ be a lifting in $\mathbb{D}$ of $\gamma_{v}$, it is clear that if $\gamma_{v}$ is bounded, then the endpoint of $\tilde{\gamma}_{v}$ has to be a point in $\Lambda_{b}(\Gamma)$, and conversely. Hence,

$$
H D(\mathscr{B}(p))=H D\left(\Lambda_{b}(\Gamma)\right) \leq H D\left(\Lambda_{c}(\Gamma)\right)=\delta(\Gamma) .
$$

We may allow elliptic elements in the Fuchsian group, and with no essential changes in the argument one also obtains in that case that $H D\left(\Lambda_{b}(\Gamma)\right)=\delta(\Gamma)$. 0.3. Some notations and basic definitions. For a Fuchsian group $\Gamma$ we shall denote the limit set and the conical limit set, respectively, by $\Lambda(\Gamma)$ and $\Lambda_{c}(\Gamma)$.

We will use $I(a, R)$ to denote the open interval of center $a$ and radius $R$. If $I=I(a, R)$ and $c>0$ we will denote $I(a, c R)$ by $c I$ and furthermore, if 
$\mathscr{A}$ is a collection of intervals, $\mathscr{A}=\left\{I\left(a_{k}, R_{k}\right)\right\}$, we will denote the collection $\left\{I\left(a_{k}, c R_{k}\right)\right\}$ by $c \mathscr{A}$.

For a point $a \in \mathbb{R}$, and a positive number $R$, we shall denote by $H(a, R)$ the euclidean disk contained in $\mathbb{H}^{2}$, of euclidean radius $R$, and which is tangent to the real line at the point $a$. Such a disk is called a horodisk, and the point $a$ is its base point. The interval $I(a, r)$ is called the shadow of the horodisk $H(a, r)$.

Given a set $E, \partial E$ will denote its boundary. If $E$ is a finite set, \#E denotes the number of elements of $E$. If $(X, d)$ is a metric space, and $E \in X$, and $\alpha \in(0,1]$, we denote the Hausdorff $\alpha$-dimensional content (with respect to the metric $d$ ) of the set $E$ by $M_{\alpha}(E)$. It is defined as

$$
M_{\alpha}(E)=\inf \left\{\sum r_{i}^{\alpha}\right\},
$$

the infimum being taken over all countable coverings of $E$ by balls of radius $r_{i}$. The Hausdorff dimension (with respect to $d$ ) of the set $E$ is defined by $H D(E):=\inf \left\{\alpha: M_{\alpha}(E)=0\right\}$.

We will say that two functions $A, B$ are comparable if there exist constants $c_{1}, c_{2}$ such that $c_{1} B<A<c_{2} B$ and we will write $A \sim B$. The letters $C, c$, and $c_{i}$, will denote generic absolute constants.

\section{REDUCTION TO SURFACES OF FINITE TYPE}

Write a general Riemann surface $\mathscr{R}$ as a quotient $\mathscr{R}=\mathbb{H}^{2} / \Gamma$, where $\Gamma$ is a Fuchsian group with no elliptic elements. As we have seen in the introduction, $(0.2)$, the inequality

$$
H D(\mathscr{B}(p)) \leq \delta(\Gamma)
$$

is straightforward.

Now, we will show that the opposite inequality holds for $\mathscr{R}$, assuming that the theorem is true for Riemann surfaces of finite type.

Let $\Gamma_{n}$ be an increasing sequence of finitely generated subgroups whose union is $\Gamma$. By a theorem of Sullivan, [S1], one has that

$$
\delta(\Gamma)=\lim _{n \rightarrow \infty} \delta\left(\Gamma_{n}\right)
$$

On the other hand, it is clear that for each $n$,

$$
\Lambda_{b}\left(\Gamma_{n}\right) \subset \Lambda_{b}(\Gamma)
$$

but, we are assuming that $H D\left(\Lambda_{b}\left(\Gamma_{n}\right)\right)=\delta\left(\Gamma_{n}\right)$, since $\Gamma_{n}$ is finitely generated. One concludes, as desired, that $H D\left(\Lambda_{b}(\Gamma)\right) \geq \delta(\Gamma)$.

\section{RIEMANN SURFACES WITH NO CUSPIDAL ENDS}

We have seen in section 1 that it is enough to prove Theorem 1 under the extra assumption that $\mathscr{R}$ is of finite type. In this case the Riemann surface $\mathscr{R}$ can be split into a disjoint union (see [P3])

$$
\mathscr{R}=\mathbb{H}^{2} / \Gamma=X_{0} \cup \bigcup_{i=1}^{k} Y_{i} \cup \bigcup_{j=1}^{l} Z_{j}
$$

where $X_{0}$ is compact; each $Y_{i}$ is isometric to $S^{1} \times\left[a_{i},+\infty\right)$, for some $a_{i}>0$, with the metric $d r^{2}+\cosh ^{2} r d \theta^{2}$ and $Z_{j}$ is isometric to $S^{1} \times[\log 2 \pi,+\infty)$ with the metric $d r^{2}+e^{-2 r} d \theta^{2}$. 
The $Y_{i}$ 's are called funnels and the $Z_{j}$ 's are called cusps or cuspidal ends. Notice that the infimum of the lengths of the loops in the nontrivial free homotopy class of each cusp is zero, while on each funnel $Y_{i}$ it is equal to the length of the simple closed geodesic bounding the funnel, namely, $2 \pi \cosh a_{i}$. Also, observe that the length of the finite boundary of each cusp is 1 . This is Shimizu's lemma, see [Be].

If $\mathscr{R}$ does not have cuspidal ends, then $\mathscr{B}(p)$ is the set of geodesics which stay within a fixed bounded region in $\mathscr{R}$ which does not depend on the geodesic and which contains the point $p$. However, if $\mathscr{R}$ has cuspidal ends, then a direction $v \in S(p)$ belongs to $\mathscr{B}(p)$ if the geodesic $\gamma_{v}$ remains within a bounded region in $\mathscr{R}$ which depends on the geodesic and which contains the point $p$.

As usual we will denote by $C(\mathscr{R})$ the convex core of $\mathscr{R}$, i.e.,

$$
C(\mathscr{R})=\mathscr{R} \backslash \bigcup_{i=1}^{k} Y_{i} .
$$

Now, assume that $\mathscr{R}$ does not have cusps. Then a geodesic $\gamma_{v}$ which remains in the convex core $C(\mathscr{R})$ at all times is bounded since in this case $C(\mathscr{R})$ is compact. But $\gamma_{v}$ stays in $C(\mathscr{R})$ if and only if a lifting $\tilde{\gamma}_{v}$ of $\gamma_{v}$ to $\mathbb{H}^{2}$ stays in $C(\Lambda(\Gamma))$, the convex hull in $\mathbb{H}^{2}$ of the limit set. And therefore $H D(\mathscr{B}(p)) \geq H D(\Lambda(\Gamma))$. But since $\Gamma$ is geometrically finite and has no parabolics elements then $\Lambda(\Gamma)=\Lambda_{c}(\Gamma)$. Consequently, $H D(\mathscr{B}(p)) \geq \delta(\Gamma)$.

Notice that for Riemann surfaces of finite type with no cuspidal ends the three limit sets that we are considering actually coincide, $\Lambda(\Gamma)=\Lambda_{c}(\Gamma)=\Lambda_{b}(\Gamma)$.

\section{RIEMANN SURFACES WITH CUSPIDAL ENDS}

The previous two sections have reduced the proof of Theorem 1 to get a lower bound for $H D(\mathscr{B}(p))$ for $\mathscr{R}$ of finite type with at least one cuspidal end. But in this particular case we shall obtain a more precise result which we now describe.

For each cusp $Z_{j}$, and positive $h$, we let $Z_{j}(h)$ denote the region of $Z_{j}$ isometric to $S^{1} \times[\log 2 \pi+h,+\infty)$, which is the set of points of $Z_{j}$ whose distance from the boundary is at least $h$. From now on the distance inside a cusp from the boundary loop will be called the height into the cusp. We consider now the set $\mathscr{B}(p, h)$ of directions $v \in S(p)$ such that the geodesic $\gamma_{v}$ remains in $C(\mathscr{R}) \backslash \bigcup_{j=1}^{l} Z_{j}(h)$ at all times.

We shall obtain the following estimate on the Hausdorff dimension of the set $\mathscr{B}(p, h)$.

Theorem 2. Let $\mathscr{R}=\mathbb{H}^{2} / \Gamma$ be of finite type with at least one cuspidal end. Then there exists $c=c(\Gamma)>0$ such that for all $h>0$

$$
H D(\mathscr{B}(p, h)) \geq \delta(\Gamma)-\frac{c}{h} .
$$

Given a fixed cusp $Z$ there exists a conjugacy class of maximal cyclic parabolic subgroups of $\Gamma$, also called a cusp, which contains a subgroup of $\Gamma$ generated by a parabolic element $\gamma$, say, with fixed point $\xi$ in the limit set of $\Gamma$. Besides, there exists a Möbius transformation $A$ such that $A(\infty)=\xi$ and $A^{-1} \circ \gamma \circ A$ 
is the translation $z \longmapsto z+1$. Also, there exists a half plane

$$
U=\{z \in \mathbb{C}: \operatorname{Im} z>1\}
$$

verifying that the image of $A(U)$ under $\pi: \mathbb{H}^{2} \longrightarrow \mathbb{H}^{2} / \Gamma$, the canonical projection, is homeomorphic to $Z$ [K, p. 52].

By a theorem of Shimizu $[K$, p. 60] we have that the set

$$
\left\{g(U): g \in A^{-1} \circ \Gamma \circ A \backslash\{\mathrm{id}\}\right\}
$$

consists of a pairwise disjoint and countable family of euclidean balls contained in $\mathbb{H}^{2}$ with euclidean diameter at most 1 . These balls are tangent to $\mathbb{R}$ at certain base-points $a_{i}$; these points are the parabolic fixed points which are fixed by the elements in the conjugacy class in $A^{-1} \circ \Gamma \circ A$ of the translation $z \longmapsto z+1$. Also, notice that $a_{i}=\left(A^{-1} \circ \gamma_{i} \circ A\right)(\infty)$ with $\gamma_{i} \in \Gamma \backslash \Gamma_{\xi}$ and $\Gamma_{\xi}=\{\gamma \in \Gamma: \gamma(\xi)=\xi\}$.

Henceforth we will refer to these balls as the horodisks corresponding to the cusp $Z$. Recall the notations, (0.3), for horodisks $H(a, R)$, and their shadows $I(a, R)$.

For all $t>0$ we will denote by $A(t)$ the set of points $\xi \in \Lambda(\Gamma)$ satisfying

$$
\left|\xi-a_{i}\right|>t R_{i}
$$

for all the horodisks $H\left(a_{i}, R_{i}\right)$ corresponding to all the cusps.

The set $\bigcup_{t>0} A(t)$ is the set of badly approximable points by parabolic fixed points, and Theorem 2 follows from the following estimate on the size of each particular $A(t)$.

Theorem 3. Let $\mathscr{R}=\mathbb{H}^{2} / \Gamma$ be a Riemann surface of finite type with at least one cuspidal end. Then for $\varepsilon \leq \varepsilon_{0}$,

$$
M_{\delta(\Gamma)-\varepsilon}(A(t))>0
$$

with $\varepsilon=\frac{c}{|\log t|}$ and $c$ a positive constant. In particular,

$$
H D(A(t)) \geq \delta(\Gamma)-\varepsilon
$$

Here we use $M_{\alpha}$, with $0<\alpha \leq 1$, to denote $\alpha$-dimensional Hausdorff content, see $(0.3)$.

Theorem 2 follows from Theorem 3 and the following geometric observation (see [S2]): we may identify the set $A(c t)$ with a subset of $\mathscr{B}\left(p, \log \frac{1}{t}\right)$ in a smooth manner. This is done as follows. Let $\gamma_{v}$ be a geodesic on $\mathscr{R}$ and $\tilde{\gamma}_{v}$ be a lifting of $\gamma_{v}$ to $\mathbb{H}^{2}$. Let $x \in \mathbb{R}$ be the endpoint of $\gamma_{v}$ and let $H(a, R)$ be a horodisk associated to some cusp $Z_{j}$. Denote by $t_{1}$ the entry time of $\tilde{\gamma}_{v}$ in $H(a, R)$ and $t_{2}$ the exit time. Then if $|x-a|=r \leq R$, then the maximum height attained by $\gamma_{v}$ into the cusp $Z_{j}$ between times $t_{1}$ and $t_{2}$ is comparable to $\log \frac{R}{r}$. Now, if $x \in A(c t)$ then the maximum height attained in all cusps is finite, and since $x \in \Lambda(\Gamma)$ then the geodesic $\gamma_{v}$ remains in $C(\mathscr{R}) \backslash \bigcup_{j=1}^{l} Z_{j}(h)$ for some $h$.

The key of the proof of Theorem 3 is the construction of certain Cantor-like sets contained in $\bigcup_{t>0} A(t)$. The description of these Cantor sets is the content of the following lemma. 
Lemma 1. There exist $0<\rho<\frac{1}{2}, c>0$ and $n_{1} \in \mathbb{N}$ such that for all $n \geq n_{1}$ and $t=\rho^{n}$, there exists a Cantor set $C_{t}$ with the following properties:

(i) $C_{t}=\bigcap_{k=0}^{\infty} \bigcup_{I_{k} \in \mathscr{I}_{k}} I_{k}$ where $\mathscr{J}_{0}=\left\{I_{0}=I\left(a, r_{0}\right)\right\}$ with $r_{0}>t$ and in general each $\mathscr{I}_{k}$ is a finite collection of disjoint intervals on $\mathbb{R}$ with radii comparable to $r_{k}=t r_{k-1}$.

(ii) If $I(a, r) \in \mathscr{I}_{j}$, then the center a is the base point of some horodisk $H(a, R)$ corresponding to a fixed cusp $Z$ and the radius $r=s R$ with $s$ a positive constant. Moreover, $r \sim t r^{\prime}$ where $r^{\prime}$ is the radius of some interval of the family $\mathscr{I}_{j-1}$.

(iii) There exists a constant $c^{\prime}, 0<c^{\prime}<1$, such that each interval in $\mathscr{J}_{j}$ is contained in some interval of $c^{\prime} \mathscr{I}_{j-1}$.

(iv) For all $j$, every interval of $\mathscr{I}_{j}$ contains the same number of intervals of $\mathscr{F}_{j+1}$. This number is comparable to $1 / t^{\delta(\Gamma)}$.

Finally,

(v) $A(c t) \supset C_{t}$.

(vi) $M_{\delta(\Gamma)-\varepsilon}\left(C_{t}\right)>0$, if $\varepsilon=\frac{c}{|\log t|}$.

We also need some properties of the Patterson measure $\mu$, see [P3], [S1], and also $[\mathrm{N}]$, which is a canonical $\Gamma$-conformal probability measure of exponent $\delta=\delta(\Gamma)$ supported on the limit set. We recall that $\mu$ being a $\Gamma$-conformal measure means that $\mu$ is finite on bounded sets and satisfies

$$
\mu(\gamma(X))=\int_{X}\left|\gamma^{\prime}\right|^{\delta} d \mu
$$

for all $\gamma \in \Gamma$ and all measurable $X \subset \Lambda(\Gamma)$. We recall also, see [N], that if $\Gamma$ is geometrically finite then the Patterson measure is atomless.

In the next lemma we record a precise estimate, due to Sullivan and Tukia, see [S3] and [Tu1], of the value of $\mu$ on some intervals.

Lemma 2. Let $\xi \in \Lambda(\Gamma)$, then

$$
\mu(I(\xi, r)) \sim r^{\delta(\Gamma)} \Phi((\xi, r))
$$

where $\Phi((\xi, r)) \sim\left(\frac{R}{r}\right)^{1-\delta}$ if the point $(\xi, r) \in \mathbb{H}^{2}$ belongs to a horodisk of radius $R$ corresponding to a cusp. Otherwise $\Phi((\xi, r)) \sim 1$.

Remark. There is an anologous statement in higher dimensions. Specifically, if the point $(\xi, r) \in \mathbb{H}^{d+1}$ belongs to a horoball $H(a, R)$ associated to a cusp $Z$ of rank $k$, then $\Phi((\xi, r)) \sim\left(\frac{R}{r}\right)^{k-\delta}$. Otherwise, $\Phi((\xi, r)) \sim 1$.

Proof of Theorem 3. Theorem 3 follows inmediately from properties (v) and (vi) of the Cantor set described in Lemma 1. We show now how these two properties are obtained and postpone the construction of the Cantor set to the next section.

Proof of (v) of Lemma 1. Notice that we have fixed a cusp $Z$, and that at first sight it appears as if only information about height into that specific cusp could be obtained.

To prove that $C_{t} \subset A(c t)$, we let $\xi \in C_{t}$; then for each $j=0,1,2, \cdots$ there exists a horodisk $H\left(a_{j}, R_{j}\right)$, say, associated to $Z$, such that $\left|\xi-a_{j}\right|<c_{0} r_{j}$ with $r_{j} \sim R_{j}$ and $c_{0}$ a positive constant. 
An elementary geometric argument shows that for two disjoint horodisks $H(a, R)$ and $H(b, S)$ one has

$$
|a-b| \geq 2 R^{\frac{1}{2}} S^{\frac{1}{2}} \text {. }
$$

Now, let $H(a, R)$ be a horodisk corresponding to some cusp, not necessarily to the one we have fixed in the construction of $C_{t}$, namely, $Z$. We must show that

$$
|\xi-a|>c t R
$$

Using (3.1) we get that

$$
|\xi-a| \geq\left|a-a_{j}\right|-\left|\xi-a_{j}\right| \geq 2 R^{\frac{1}{2}} R_{j}^{\frac{1}{2}}-c_{0} r_{j} .
$$

Since $r_{j} \sim R_{j}$ for all $j$ we have that there exist absolute constants $c_{1}, c_{2}>0$ such that $c_{1} r_{j} \leq R_{j} \leq c_{2} r_{j}$. First, we notice that if $R \geq \frac{c_{0}^{2} r_{0}}{c_{1}}$, then by (3.3) for $j=0$ we have $|\xi-a|>c_{0} r_{0}>c_{0} t \geq c_{0} t R$, since $R \leq 1$. This gives (3.2).

On the other hand, if $R<\frac{c_{0}{ }^{2} r_{0}}{c_{1}}$, then since the $r_{j}$ 's tend to 0 , there exists $j \geq 1$ such that $c_{0}^{2} r_{j} \leq c_{1} R<c_{0}^{2} r_{j-1}$. Then, using that $c_{1} r_{j} \leq R_{j}(\xi)$ and that $R \geq \frac{c_{0}^{2} r_{j}}{c_{1}}$ in (3.3) we get that $|\xi-a| \geq c_{0} r_{j}$. Finally, $r_{j}=t r_{j-1}>\frac{c_{1}}{c_{0}^{2}} t R$, which gives the desired inequality (3.2)

Proof of (vi) of Lemma 1. If $C_{t} \subset A(c t)$ is the Cantor set of Lemma 1, then we must show that

$$
M_{\delta(\Gamma)-\varepsilon}\left(C_{t}\right)>0
$$

with $\varepsilon=\frac{c}{|\log t|}$ and $c$ a positive constant independent of $t$. To show (3.3) we will follow a well-known idea of Frostman: we will construct a probability measure $\nu_{t}$ supported in $C_{t}$, with the property that for all intervals $I=I\left(a_{I}, r_{I}\right)$

$$
\nu_{t}(I) \leq C r_{I}^{\delta(\Gamma)-\varepsilon}
$$

with $C>0, \varepsilon=\frac{c}{|\log t|}$ and $c$ a positive constant independent of $t$.

From (3.4) it is clear that for all covering $\left\{I_{s}=I\left(a_{s}, r_{s}\right)\right\}$ of $C_{t}$ we would have that

$$
1=\nu_{t}\left(C_{t}\right) \leq \sum_{s} \nu_{t}\left(I_{s}\right) \leq C \sum_{s} r_{s}^{\delta(\Gamma)-\varepsilon}
$$

and, consequently, that $M_{\delta(\Gamma)-\varepsilon}\left(C_{t}\right)>0$.

We will define $\nu_{t}$ on the intervals in the successive generations $\left\{\mathscr{J}_{k}\right\}$ inductively. First, $\nu_{t}\left(I_{0}\right)=1$ and then

$$
\nu_{t}\left(I_{k+1}\right)=\nu_{t}\left(I_{k+1} \cap C_{t}\right):=\frac{1}{\frac{\# I_{k+1}}{\# I_{k}}} \nu_{t}\left(I_{k}\right), \quad \forall I_{k+1} \in \mathscr{I}_{k+1} .
$$

And for any set $U \subset \mathbb{R}$

$$
\nu_{t}(U):=\inf \sum_{F \in \mathscr{F}} \nu_{t}(F)
$$

where the infimum is taken over all the coverings $\mathscr{F}$ of $U$ with intervals in $\bigcup_{k \geq 0} \mathscr{I}_{k}$. 
Since $\frac{\# \mathscr{I}_{k+1}}{\# \mathscr{Y}_{k}} \sim \frac{1}{t^{\delta(\Gamma)}}$ we have that $\nu_{t}\left(I_{k+1}\right) \sim t^{\delta(\Gamma)} \nu_{t}\left(I_{k}\right)$.

To verify (3.4) we assume first that $I=I_{k} \in \mathscr{I}_{k}$. Then, there exist $c_{1}, c_{2}>$ 0 , independent of $t$, such that $\nu_{t}\left(I_{k}\right) \leq\left(c_{1} t^{\delta(\Gamma)}\right)^{k}$ and $r_{I} \geq\left(c_{2} t\right)^{k}$. It is not difficult to see that taking $t=\rho^{n}$ small enough, i.e., $n$ large enough, we have that for $\varepsilon \geq \frac{\delta(\Gamma) \log c_{2}-\log c_{1}}{\log t c_{2}} \geq \frac{c}{|\log t|}$

$$
\nu_{t}\left(I_{k}\right) \leq\left(c_{1} t^{\delta(\Gamma)}\right)^{k} \leq\left(c_{2} t\right)^{k(\delta(\Gamma)-\varepsilon)} \leq r_{I}^{\delta(\Gamma)-\varepsilon}
$$

Now, let us assume that $I \neq I_{k}$ for all $I_{k} \in \mathscr{I}_{k}$ and all $k=0,1,2, \cdots$. If $I \not \subset I_{0}$ and $I \cap C_{t} \neq \varnothing$, then since $C_{t} \subset c^{\prime} I_{0}$ we have that $2 r_{I} \geq\left(1-c^{\prime}\right) r_{0}$. So, if we have that $\nu_{t}\left(c^{\prime} I_{0}\right) \leq C\left(c^{\prime} r_{0}\right)^{\delta(\Gamma)-\varepsilon}$, then we also get

$$
\nu_{t}(I) \leq \nu_{t}\left(c^{\prime} I_{0}\right) \leq C\left(c^{\prime} r_{0}\right)^{\delta(\Gamma)-\varepsilon} \leq C^{\prime} r_{I}^{\delta(\Gamma)-\varepsilon} \text {. }
$$

Hence, we may assume $I \subset c^{\prime} I_{0}$.

Let $k=k(I)=\max \left\{j: I \subset I_{j}\right.$ for some $\left.I_{j} \in \mathscr{J}_{j}\right\}$. We can assume without loss of generality that $I$ intersects at least two intervals of the generation of $c^{\prime} \mathscr{I}_{k+1}$ and so there exists $c_{3}, c_{4}>0$ such that $c_{3} r_{k+1} \leq r_{I} \leq c_{4} r_{k}$. Since $r_{k+1}=t r_{k}$, we get that $c_{3} t r_{k} \leq r_{I} \leq c_{4} r_{k}$.

We have that

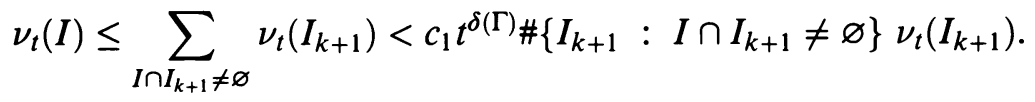

By comparing $\mu$-measures we have that

$$
\#\left\{I_{k+1}: I \cap I_{k+1} \neq \varnothing\right\} \mu\left(I_{k+1}\right) \leq \mu\left(I_{k}\right) .
$$

From Lemma 1 we know that each $I_{j} \in \mathscr{F}_{j}, j=1,2, \cdots$, is centered in a parabolic fixed point and $r_{j}$ is comparable to the radii of the horodisk with the same base point corresponding to the cusp $Z$. So, the $\mu$-measure of each $I_{j}$ is comparable to the $\mu$-measure of the corresponding horodisk. Using that $r_{j} \sim t r_{j-1}$ and Lemma 2,

$$
\mu\left(I_{k+1}\right) \sim r_{k+1}^{\delta(\Gamma)} \sim\left(t r_{k}\right)^{\delta(\Gamma)} \sim t^{\delta(\Gamma)} \mu\left(I_{k}\right) .
$$

Therefore

$$
\#\left\{I_{k+1}: I \cap I_{k+1} \neq \varnothing\right\} \leq \frac{C^{\prime \prime}}{t^{\delta(\Gamma)}}
$$

and so we get that

$$
\nu_{t}(I) \leq c_{1} C^{\prime \prime} \nu_{t}\left(I_{k+1}\right) \leq C^{\prime} r_{k+1} \delta(\Gamma)-\varepsilon \leq C r_{I} \delta(\Gamma)-\varepsilon
$$

\section{Construction of Cantor sets. Proof of Lemma 1}

The construction of the Cantor sets of Lemma 1 requires the next two lemmas. The first one concerns the equidistribution of horodisks corresponding to a single cusp and it is akin to a local version of Proposition 4 in [S2]. 
Lemma 3. There exist $\rho \in\left(0, \frac{1}{2}\right), s \geq 1$ such that for each cusp $Z_{l}$ we can find a horodisk $H_{l}^{\prime}=H\left(a_{l}^{\prime}, R_{l}^{\prime}\right)$ associated to $Z_{l}$ with the property that: for every $n \geq n_{0}=n_{0}(\Gamma, s)$ there is a collection $T_{n}\left(Z_{l}\right)$ of horodisks corresponding to this cusp $Z_{l}$ such that

(i) if $H(a, R) \in T_{n}\left(Z_{l}\right)$, then $a \in I\left(a_{l}^{\prime}, s R_{l}^{\prime}\right)$ and $R \in\left[\rho^{n+1}, \rho^{n}\right)$.

(ii) $\# T_{n}\left(Z_{l}\right) \sim\left(1 / \rho^{n}\right)^{\delta}$.

Proof. See appendix.

Notice that there exists $c_{1}>0$ such that for $n$ large enough we have

$$
\sum_{H(a, R) \in T_{n}\left(Z_{l}\right)} \mu(I(a, R)) \leq c_{1} \mu\left(I\left(a_{l}^{\prime}, s R_{l}^{\prime}\right)\right) .
$$

Besides,

$$
\sum_{H(a, R) \in T_{n}\left(Z_{l}\right)} \mu(I(a, R)) \sim \# T_{n}\left(\mathscr{E}_{l}\right)\left(\rho^{n}\right)^{\delta(\Gamma)} .
$$

Hence, Lemma 3 is telling us that up fixed constants the number of horodisks of $T_{n}\left(Z_{l}\right)$ is maximal.

We will also need the following result in the proof of Lemma 1.

Lemma 4. Let $H_{1}=H\left(a_{1}, R_{1}\right)$ and $H_{2}=H\left(a_{2}, R_{2}\right)$ be two horodisks corresponding to the same cusp $Z$. Then, given $C \geq 1$, there exists $\gamma_{1}, \gamma_{2} \in \Gamma$ such that

(i) $\quad \gamma_{i}\left(H_{1}\right)=H_{2}, \quad i=1,2$,

(ii) $\frac{1}{3 C} \frac{R_{2}}{R_{1}} \leq\left|\gamma_{1}^{\prime}(z)\right| \leq 3 \frac{R_{2}}{R_{1}}, \quad$ for $\operatorname{Re}\left(z-a_{1}\right) \geq 0, \quad\left|z-a_{1}\right| \leq C R_{1}$,

(iii) $\frac{1}{3 C} \frac{R_{2}}{R_{1}} \leq\left|\gamma_{2}^{\prime}(z)\right| \leq 3 \frac{R_{2}}{R_{1}}, \quad$ for $\operatorname{Re}\left(z-a_{1}\right) \leq 0, \quad\left|z-a_{1}\right| \leq C R_{1}$.

Proof. Since $H_{1}$ and $H_{2}$ correspond to the same cusp we know that there exists $\tilde{\gamma} \in \Gamma$ such that $\tilde{\gamma}\left(H_{1}\right)=H_{2}$. Besides it is clear, see, e.g., [K, p. 60], that there exist $\tilde{\gamma}_{1}, \tilde{\gamma}_{2}$ in the stabilizer of $a_{2}$ such that $\gamma_{i}=\tilde{\gamma}_{i} \circ \tilde{\gamma} \in \Gamma$ satisfy

(i) $\gamma_{i}\left(H_{1}\right)=H_{2}, \quad i=1,2$,

(ii) $\gamma_{i}\left(a_{1}+2 R_{1} \sqrt{-1}\right)=w_{i}$ with $w_{i} \in \partial H_{2}$ such that the hyperbolic length of the portion of $\partial H_{2}$ between $w_{i}$ and $a_{2}+2 R_{2} \sqrt{-1}$ is less than or equal to $1(i=1,2)$. Moreover, $\operatorname{Re}\left(w_{1}-a_{2}\right) \geq 0$ and $\operatorname{Re}\left(w_{2}-a_{2}\right) \leq 0$.

Let us denote by $H_{0}$ the horocyle $H_{0}=H(0,1)$. We now show that if $\eta$ is a Möbius transformation of $\mathbb{H}^{2}$,

(i') $\eta\left(H_{0}\right)=H_{0}$,

(ii') $\eta(2 \sqrt{-1})=w$, where the hyperbolic length of the portion of $H_{0}$ between $2 \sqrt{-1}, w$ is less than or equal to 1 and $\operatorname{Re}(w) \geq 0$.

Then $1 /(3 C) \leq\left|\eta^{\prime}(z)\right| \leq 3$ for $|z| \leq C$ and $\operatorname{Re}(z) \geq 0$.

The desired result for $\gamma_{1}$ is then obtained by composing $\eta$ with translations and dilations. The argument for $\gamma_{2}$ is similar. 
Let $\eta(z)=\frac{a z+b}{c z+d}$ with $a d-b c=1$. Since $\eta(0)=0$ we know that $b=0$. Let $w=x_{0}+y_{0} \sqrt{-1}$, using that $\eta(2 \sqrt{-1})=w$ we get

$$
\left\{\begin{array}{l}
x_{0}=\frac{4 c}{d\left(4 c^{2}+d^{2}\right)}, \\
y_{0}=\frac{2}{4 c^{2}+d^{2}} .
\end{array}\right.
$$

From (ii') one deduces that $0 \leq x_{0} \leq 1$ and $1 \leq y_{0} \leq 2$. Hence we get

$$
\left\{\begin{array}{l}
0 \leq 4 \frac{c}{d} \leq 4 c^{2}+d^{2} \\
1 \leq 4 c^{2}+d^{2} \leq 2
\end{array}\right.
$$

and, consequently, $0 \leq c / d \leq 1 / 2$ and $1 / 2 \leq d^{2} \leq 2$.

Therefore for $z=\bar{x}+y \sqrt{-1}$ with $|z| \leq \bar{C}$ and $x \geq 0$ we have that

$$
\frac{1}{2} \leq c^{2}|z|^{2}+d^{2}+2 c d x \leq \frac{9}{2} C^{2}
$$

and since

$$
\left|\gamma^{\prime}(z)\right|^{2}=\frac{1}{|c z+d|^{2}}=\frac{1}{c^{2}|z|^{2}+d^{2}+2 c d x}
$$

we get the result.

We are now ready to give the proof of Lemma 1.

Proof of Lemma 1. Let $s$ and $n_{0}(\Gamma, s)$ as in Lemma 3. We fix a horodisk, $H_{0}=H\left(a_{0}, R_{0}\right)$, which corresponds to the cusp $Z$.

Let $H^{\prime}=H\left(a^{\prime}, R^{\prime}\right)$ be the horodisk corresponding to $Z$ given in Lemma 3 . This $H^{\prime}$ will play the role of a reference unit in the construction.

We start by defining $\mathscr{H}_{0}=\left\{H_{0}\right\}, \mathscr{J}_{0}=\left\{I_{0}=I\left(a_{0}, r_{0}\right)\right\}$, where $r_{0}=s R_{0}$.

For the first generation we use the family $T_{n}=T_{n}(Z)\left(n \geq n_{0}\right)$ from Lemma 3. Without loss of generality we can suppose that half of the intervals of the family $T_{n}$ have centers in $\left[a^{\prime}, \infty\right)$. Moreover, by Lemma 4 there exists $\gamma \in \Gamma$ such that

(1) $\gamma\left(H^{\prime}\right)=H_{0}$

(2) $\frac{1}{6 s} \frac{R_{0}}{R^{\prime}} \leq\left|\gamma^{\prime}(z)\right| \leq 3 \frac{R_{0}}{R^{\prime}}$, for $\left|z-a^{\prime}\right|<(2 s) R^{\prime}, \operatorname{Re}\left(z-a^{\prime}\right) \geq 0$.

Notice that if $H(\tilde{a}, \tilde{R})=\gamma\left(H(a, R)\right.$ for some $H(a, R) \in T_{n}$ with $a \in$ $\left[a^{\prime}, \infty\right)$, then $R \sim \frac{R_{0}}{R^{\prime}} \rho^{n+1}$. Hence $R \sim R_{0} t$ with $t=\rho^{n+1}$.

Now, for $n$ large enough it is clear that there is a subcollection $\tilde{T}_{n} \subset T_{n}$ such that the set $\mathscr{H}_{1}=\left\{\gamma(H(a, R)): H(a, R) \in \tilde{T}_{n}\right\}$ satisfies

(i) For all $H_{i}=H\left(a_{i}, R_{i}\right), H_{j}=H\left(a_{j}, R_{j}\right) \in \mathscr{H}_{1}, I\left(a_{i}, s R_{i}\right) \cap I\left(a_{j}, s R_{j}\right)$ $=\varnothing$.

(ii) There exists $0<c^{\prime}<1$ such that if $H(a, R) \in \mathscr{H}_{1}$, then $I(a, s R) \subset$ $I\left(a_{0}, c^{\prime} s R_{0}\right)$.

(iii) If $H(a, R) \in \mathscr{H}_{1}$ then $R \sim R_{0} t$ with $t=\rho^{n+1}$.

(iv) $\# \mathscr{H}_{1} \sim\left(1 / \rho^{n+1}\right)^{\delta}$.

Finally, we let

$$
\mathscr{J}_{1}=\left\{I(a, s R): H(a, R) \in \mathscr{H}_{1}\right\}
$$


By construction, it is clear that the intervals of $\mathscr{I}_{1}$ are disjoint with radii comparable to $r_{1}:=r_{0} t$ and that $\mathscr{I}_{1} \subset c^{\prime} \mathscr{I}_{0}$. Besides,

$$
\# \mathscr{I}_{1}=\# \mathscr{H}_{1} \sim \frac{1}{t^{\delta(\Gamma)}} .
$$

Now, let us assume that we have reached the $j$-th stage and that we have already constructed $\mathscr{H}_{1}, \cdots, \mathscr{H}_{j}, \mathscr{I}_{1}, \cdots, \mathscr{I}_{j}$ and that $r_{j}$ has been defined. Let us number the horodisks in $\mathscr{H}_{j}$ as $H_{1}, \cdots, H_{k}$ with respective radii $R_{1}, \cdots$, $R_{k}$. By Lemma 4 , for each $H_{u} \in \mathscr{H}_{j}$ there exists $\gamma_{u} \in \Gamma$ such that

(1') $\gamma_{u}\left(H^{\prime}\right)=H_{u}$,

(2') $\frac{1}{6 s} \frac{R_{u}}{R^{\prime}} \leq\left|\gamma_{u}^{\prime}(z)\right| \leq 3 \frac{R_{u}}{R^{\prime}}, \quad$ for $\left|z-a^{\prime}\right|<R^{\prime}, \operatorname{Re}\left(z-a^{\prime}\right) \geq 0$.

For each $u$, and using the $\gamma_{u}$ 's we first define the family of horodisks

$$
\mathscr{H}_{j+1, u}=\left\{\gamma_{u}(H(a, R)): H(a, R) \in \mathscr{H}_{1}\right\} .
$$

If $H(a, R) \in \mathscr{H}_{j+1, u}$, then $R \sim \frac{R_{s}}{R^{\prime}} \rho^{n+1} \sim r_{j} t$. Besides $\# \mathscr{H}_{j+1, s}=\# H_{1} \sim \frac{1}{t^{\delta(\Gamma)}}$. The union of these collections of horodisks is the family $\mathscr{H}_{j+1}$, namely, $\mathscr{H}_{j+1}=$ $\bigcup_{u=1}^{k} \mathscr{H}_{j+1, u}$.

And finally, we define

$$
\mathscr{J}_{j+1}=\left\{I(a, s R): H(a, R) \in \mathscr{H}_{j+1}\right\} \text {. }
$$

By construction it is again clear that the intervals of the family $\mathscr{I}_{j+1}$ are disjoint with radii comparable to $r_{j+1}:=r_{j} t$ and that $\mathscr{J}_{j+1} \subset c^{\prime} \mathscr{F}_{j}$.

This finishes the inductive step of the construction of the set $C_{t}$.

\section{HYPERBOLIC 3-MANIFOLDS}

The proof of Theorem $1^{\prime}$ is similar to the proof of Theorem 1 except that now when $n=3$ and there are cusps and all of non-maximal rank the proof of the crucial lemma has to be modified, requiring a new idea which we now explain.

For any geometrically finite group $\Gamma$ it is known [Tu1,Tu2] that its parabolic fixed points have cusp neighbourhoods. We recall that for any parabolic fixed point $p$, an open set $U \subset \overline{\mathbb{H}}^{3}$ is called a cusp neighbourhood of $p$ if there exists $h \in \operatorname{Möb}\left(\mathbb{H}^{3}\right)$ such that $h(p)=\infty$ and

(i) $h(U)=\overline{\mathbb{H}}^{3} \backslash\left(\mathbb{R}^{k} \times B^{3-k}\right)$ where $B^{3-k}$ is the unit ball of $\mathbb{R}^{3-k}$ and $k$ is the rank of the cusp.

(ii) $\operatorname{closure}(U) \cap \Lambda(\Gamma)=\{p\}$.

(iii) $\gamma(U)=U$ for all $\gamma \in \Gamma_{p}$ and $\gamma(U) \cap U=\varnothing$ for all $\gamma \in \Gamma \backslash \Gamma_{p}$.

Notice that $h^{-1}\left(\left\{\left(x_{1}, x_{2}, x_{3}\right) \in \mathbb{R}^{3}: x_{3} \geq 1\right\}\right)$ is the horoball with base-point $p$.

The substitute for Lemma 4 that we need is the following:

Lemma $4^{\prime}$. Let $H_{1}=H\left(a_{1}, R_{1}\right)$ and $H_{2}=H\left(a_{2}, R_{2}\right)$ be two horoballs corresponding to the same cusp $Z$. Then, given $C \geq 1$ there exists $\gamma_{1}, \gamma_{2} \in \Gamma$ such that 
(i) $\gamma_{i}\left(H_{1}\right)=H_{2}, \quad i=1,2$,

and for $x=\left(x_{1}, x_{2}, x_{3}\right) \in \mathbb{H}^{3}$,

(ii) $\frac{1}{c C} \frac{R_{2}}{R_{1}} \leq\left|\gamma_{1}^{\prime}(x)\right| \leq c \frac{R_{2}}{R_{1}}, \quad$ if $\left(x_{1}, x_{2}\right) \in Q_{1}, \quad\left|x-a_{1}\right| \leq C R_{1}$.

(iii) $\frac{1}{K C} \frac{R_{2}}{R_{1}} \leq\left|\gamma_{2}^{\prime}(x)\right| \leq K \frac{R_{2}}{R_{1}}$, if $\left(x_{1}, x_{2}\right) \in Q_{2}, \quad\left|x-a_{1}\right| \leq C R_{1}$.

Here $K$ is a positive constant and $Q_{1}, Q_{2}$ denote the two complementary half planes in $\mathbb{R}^{2}$ of a line in $\mathbb{R}^{2}$ through $a_{1}$.

Proof. Let $q$ be the rank of the cusp. As remarked above cusps of maximal rank $(q=2)$ are handled as in the proof of Lemma 4.

Let $q=1$. Without loss of generality we can suppose that $Z$ is the cusp corresponding to the transformation $\left(x_{1}, x_{2}, x_{3}\right) \longrightarrow\left(x_{1}+1, x_{2}, x_{3}\right)$ and $U_{\infty}:=\overline{\mathbb{H}}^{3} \backslash\left(\mathbb{R} \times B^{2}\right)$ is the cusp neighbourhood at infinity.

Since $H_{1}$ and $H_{2}$ correspond to the same cusp $Z$, we have that there exist $\tilde{\gamma}_{i} \in \Gamma(i=1,2)$ such that

$$
H_{i} \subset U_{i}:=\tilde{\gamma}_{i}\left(U_{\infty}\right)
$$

with $U_{i}$ a cusp neighbourhood at the base point $a_{i}$ of the horoball $H_{i}$. Let us denote by $N_{i}$ the "north" pole of $H_{i}$ ( $a_{i}$ is the south pole).

The set $\tilde{\gamma}_{i}\left(U_{i}\right)$ is the convex hull in $\overline{\mathbb{H}}^{3}$ of two disks $B_{i j}=B\left(c_{i j}, R_{i j}\right)$ $(j=1,2)$ in $\mathbb{R}^{2}$ such that $\bar{B}_{i 1} \cap \bar{B}_{i 2}=\left\{a_{i}\right\}$. Let $C_{i}$ be the geodesic in $\mathbb{H}^{3}$ of maximum euclidean length with end-points in $\partial B_{i 1} \cup \partial B_{i 2} . C_{i}$ and $H_{i}$ are tangent; let the point of tangency be denoted by $p_{i}$, then it is geometrically clear that the hyperbolic distance from the north pole $N_{i}$ of $H_{i}$ to $p_{i}$ is at most 1 .

Notice that $q_{i}:=\gamma_{i}^{-1}\left(p_{i}\right)=\left(t_{i}, 0,1\right)$ for some $t_{i} \in \mathbb{R}$. Let $\omega$ be the transformation $\left(x_{1}, x_{2}, x_{3}\right) \longrightarrow\left(x_{1}+1, x_{2}, x_{3}\right) \in \Gamma$. Consider $\eta_{k}=\tilde{\gamma}_{2} \omega^{k} \tilde{\gamma}_{1}^{-1}$, $\forall k \in \mathbb{Z}$. Observe that $\eta_{k}\left(H_{1}\right)=H_{2}$ and $\eta_{k}\left(a_{1}\right)=a_{2}$. The important fact is that for each $k$ the point $\eta_{k}^{-1}\left(N_{2}\right)$ lies in $\tilde{\gamma}_{1}\left(L_{1}\right)$ where $L_{1}$ is the line in $x_{3}=1$ through $\tilde{\gamma}_{2}^{-1}\left(N_{2}\right)$ which is parallel to $\left\{x_{2}=0, x_{3}=1\right\}$. Similarly, for all $k$ the point $\eta_{k}\left(N_{1}\right)$ lies in $\tilde{\gamma}_{2}\left(L_{2}\right)$ where $L_{2}$ is the line parallel to $L_{1}$ through $\tilde{\gamma}_{1}^{-1}\left(N_{1}\right)$. Using that $\operatorname{dist}\left(N_{i}, p_{i}\right) \leq 1$ for $i=1,2$ one gets that $\operatorname{dist}\left(L_{1}, L_{2}\right) \leq 2$.

Let $k_{0} \in \mathbb{Z}$ be such that $x_{3}\left(\eta_{k_{0}}{ }^{-1}\left(N_{2}\right)\right)$ is maximum, i.e., $\operatorname{dist}\left(\eta_{k_{0}}{ }^{-1}\left(N_{2}\right), N_{1}\right)$ is minimum. Since $\operatorname{dist}\left(L_{1}, L_{2}\right) \leq 2$ we have that $\operatorname{dist}\left(\eta_{k_{0}}^{-1}\left(N_{2}\right), N_{1}\right) \leq 3$. 1,2 .

Now, let $\gamma_{1}=\eta_{k_{0}+2}$ and $\gamma_{2}=\eta_{k_{0}-2}$. Then $\operatorname{dist}\left(\gamma_{i}\left(N_{1}\right), N_{2}\right) \leq 5$ for $i=$

It is geometrically clear that

$$
\left|\gamma_{1}^{-1}(\infty)-\gamma_{2}^{-1}(\infty)\right|>c_{0}^{\prime} R_{1} \text { and } c_{1}^{\prime} R_{1} \leq\left|\gamma_{i}^{-1}(\infty)\right| \leq c_{2}^{\prime} R_{1} \quad(i=1,2)
$$

for some positive constants $c_{0}^{\prime}, c_{1}^{\prime}, c_{2}^{\prime}$. Take $L$ to be the line through $a_{1}$ and the midpoint of the segment joining $\gamma_{1}^{-1}(\infty)$ and $\gamma_{2}^{-1}(\infty)$, and $Q_{1}, Q_{2}$ to 
be the complementary half planes of the line $L$ such that $\gamma_{i}{ }^{-1}(\infty) \in Q_{i} \quad(i=$ $1,2)$. Then for all $x \in Q_{i} \quad(i=1,2)$, we have that $\left|x-\gamma_{j}^{-1}(\infty)\right| \geq c_{0} R_{1}$ for $j \in\{1,2\}$ and $j \neq i$. Here $c_{0}$ is also a positive constant.

Now, let us denote by $H_{0}$ the horocycle $H_{0}=H(0,1)$. Let $\eta$ be a Möbius transformation of $\mathbb{H}^{3}$ such that

(i') $\eta\left(H_{0}\right)=H_{0}$.

(ii') $\eta((0,0,2))=\tilde{x}$, where the hyperbolic distance between $(0,0,2)$ and $\tilde{x}$ is less than or equal to 5 .

For $x \in \mathbb{H}^{3}$ such that $|x| \leq C$ and $\left|x-\eta^{-1}(\infty)\right| \geq c_{0}$, we will show that

$$
\frac{1}{K C} \leq\left|\eta^{\prime}(x)\right| \leq K, \quad \text { for an absolute constant } K>0 .
$$

The desired result for $\gamma_{i}(i=1,2)$, is then obtained by composing $\eta$ with translations and dilations.

In order to get (5.1) we will use the representation by quaternions of the Mobius transformations acting on $\mathbb{H}^{3}$ (see, e.g., [A], [B] for details). We will identify $\left(x_{1}, x_{2}, x_{3}\right) \in \mathbb{H}^{3}$ with the quaternion $x_{1}+x_{2} \mathbf{i}+x_{3} \mathbf{j}$.

For all $x=x_{1}+x_{2} \mathbf{i}+x_{3} \mathbf{j}$ we have that

$$
\eta(x)=\frac{a x+b}{c x+d}
$$

with $a, b, c, d \in \mathbb{C}$ and $a d-b c=1$.

Since $\eta(0)=0$ we know that $b=0$. Let $\tilde{x}=\tilde{x}_{1}+\tilde{x}_{2} \mathbf{i}+\tilde{x}_{3} \mathbf{j}$. Using that $\eta(2 \mathbf{j})=\tilde{x}$ we get

$$
\left\{\begin{array}{l}
\tilde{x}_{1}=\frac{4 \operatorname{Re}(\bar{c} \bar{d})}{|d|^{2}\left(4|c|^{2}+|d|^{2}\right)}, \\
\tilde{x}_{2}=\frac{4 \operatorname{Im}(\bar{c} \bar{d})}{|d|^{2}\left(4|c|^{2}+|d|^{2}\right)}, \\
\tilde{x}_{3}=\frac{2}{4|c|^{2}+|d|^{2}} .
\end{array}\right.
$$

From (ii') we have that $c^{\prime} \leq \tilde{x}_{3} \leq 2$, for some $c^{\prime}>0$, and $|\tilde{x}| \leq 1$. Moreover, from the equalities for $\tilde{x}_{1}, \tilde{x}_{2}$ one deduces that

$$
\frac{|c|}{|d|}=\frac{\left(\tilde{x}_{1}^{2}+\tilde{x}_{2}^{2}\right)^{1 / 2}}{2 \tilde{x}_{3}} \text {. }
$$

Hence we get

$$
\left\{\begin{array}{l}
\frac{|c|}{|d|} \leq \frac{1}{2 c^{\prime}}, \\
1 \leq 4|c|^{2}+|d|^{2} \leq \frac{2}{c^{\prime}}
\end{array}\right.
$$

and, consequently, $|c| \leq c_{1}|d|$ and $c_{2} \leq|d| \leq c_{3}$ for some positive constants $c_{1}, c_{2}, c_{3}$.

Therefore for $x=x_{1}+x_{2} \mathbf{i}+x_{3} \mathbf{j}$ with $|x| \leq C$ and $\left|x+\frac{d}{c}\right|=\left|x-\eta^{-1}(\infty)\right| \geq c_{0}$ we have that: If $C|c| \leq \frac{|d|}{2}$, then

$$
|c x+d| \geq|d|-C|c| \geq \frac{|d|}{2} \geq \frac{c_{2}}{2} .
$$


If $C|c|>\frac{|d|}{2}$, then

$$
|c x+d|=|c|\left|x+\frac{d}{c}\right| \geq c_{0}|c| \geq \frac{c_{0}|d|}{2 C} \geq \frac{c_{0} c_{2}}{2 C} .
$$

Moreover, $|c x+d| \leq C|c|+|d| \leq\left(C c_{1}+1\right)|d| \leq\left(C c_{1}+1\right) c_{3}$.

Since $\left|\gamma^{\prime}(x)\right|=1 /|c x+d|$ we get the result.

\section{Applications}

Theorem 4. Let $\mathscr{R}$ be a complete noncompact Riemann surface with at least a cuspidal end $Z$. If $E$ is the set of directions $v \in S(p)$ such that the geodesic $\gamma_{v}$ attains a maximal height into the cusp $Z$. Then $H D(E)=1$.

Proof. We consider two different cases depending on whether $\mathscr{R}$ has Green function or not.

If $\mathscr{R}$ has Green function then it is a well-known result that given a point $p \in \mathscr{R}$ for almost all $v \in S(p)$ the geodesic $\gamma_{v}$ in $\mathscr{R}$ satisfies

$$
d_{\mathscr{R}}\left(p, \gamma_{v}(t)\right) \longrightarrow \infty \quad \text { as } t \rightarrow \infty
$$

where $d_{\mathscr{R}}$ is the distance in $\mathscr{R}$. So the result follows in this case. On the other hand, if $\mathscr{R}$ does not have Green function then $\delta(\mathscr{R})=1$, see [A], and Theorem 4 follows from Theorem 1.

For any $\lambda \in \mathbb{R}$ we denote by $\Gamma(\lambda)$ the subgroup of $\operatorname{Möb}\left(\mathbb{H}^{2}\right)$ generated by

$$
z \rightarrow z+\lambda, \quad z \rightarrow \frac{-1}{z} .
$$

These groups are called Hecke groups.

Hecke showed in $[\mathrm{H}]$ that $\Gamma(\lambda)$ is properly discontinuous if and only if

(i) $\lambda=2 \cos \frac{\pi}{q}$ with $q \in \mathbb{Z}$ and $q \geq 3$, when $\lambda<2$.

(ii) $\lambda \in \mathbb{R}$ when $\lambda \geq 2$.

In [Ro] Rosen introduced the following class of continued fractions intrinsically related to the Hecke groups,

$$
\left(m_{0}, \frac{1}{m_{1}}, \frac{1}{m_{2}}, \cdots\right):=m_{0}+\frac{1}{m_{1}+\frac{1}{m_{2}+\cdots}}
$$

where $m_{i} \in \lambda \mathbb{Z}$. These continued fractions are called $\lambda$-fractions.

In [Ro] it is shown that for $\lambda>2$ every real number in the limit set of $\Gamma(\lambda)$ may be represented uniquely as a $\lambda$-fraction. For $\lambda<2$ the notion of reduced $\lambda$-fraction is introduced and it is shown that every real number has a unique representation as a reduced $\lambda$-fraction.

The following result of Rosen describes the elements of $\Gamma(\lambda)$.

Theorem [Ro]. A transformation $T(z)=\frac{\alpha z+\beta}{\gamma z+\rho} \in \Gamma(\lambda)$ if and only if

$$
\frac{\alpha}{\gamma}=\left(m_{0}, \frac{1}{m_{1}}, \cdots, \frac{1}{m_{n}}\right)
$$

with $m_{i} \in \lambda \mathbb{Z}$, i.e., $\frac{\alpha}{\gamma}$ is a finite $\lambda$-fraction. 
Theorem 5. The Hausdorff dimension of the set of limit points $\xi \in \Lambda(\Gamma(\lambda))$ such that

$$
\left|\xi-\frac{\alpha}{\gamma}\right| \geq \frac{C(\xi)}{\gamma^{2}}
$$

for all finite $\lambda$-fractions $\frac{\alpha}{\gamma}$, is $\delta(\Gamma(\lambda))$.

Let us recall that the function $\lambda \rightarrow \delta(\Gamma(\lambda))$ is a strictly decreasing Lipschitz continuous function on $[2, \infty)$ and satisfies $\delta(\Gamma(2))=1$ and $\lim _{\lambda \rightarrow \infty} \delta(\Gamma(\lambda))=$ $\frac{1}{2}$, see, e.g., [PS].

As a final application we would like to mention a result of the first author and D. Pestana, see [FP].

Theorem [FP]. Let $\Omega$ be a planar domain, other than $\mathbb{C}, \mathbb{C} \backslash\{a\}$, and which is not conformal to $\mathbb{D} \backslash\{0\}$. Then, if $f: \mathbb{D} \longrightarrow \Omega$ is holomorphic,

$$
H D\left\{\theta: \inf _{0 \leq r<1} \operatorname{dist}\left(f\left(r e^{i \theta}\right), \partial \Omega\right)>0\right\} \geq \delta(\Omega) .
$$

The result is sharp in the sense that for each such $\Omega$ and for $f$ being a covering map one has equality in (1). Moreover for each $\eta \in[\delta(\Omega), 1]$, there is $f$ as above with the dimension in (1) equal to $\eta$.

Here dist means euclidean distance, and $\partial \Omega$ denotes the boundary of $\Omega$.

Appendix. Distribution of horodisks. Proof of Lemma 3

In this appendix we will assume that $\mathscr{R}$ has at least a cusp. Lemma 3 follows from the following result about distribution of horodisk.

Theorem 5. There exists $\rho \in\left(0, \frac{1}{2}\right)$ and a compact set $K$ of $\mathbb{R}$ such that for every cusp $Z$ the set $A_{n}(Z)$ of horodisks corresponding to this cusp with base points in $K$ and radii $R \in\left[\rho^{n+1}, \rho^{n}\right)$ satisfies for all $n \geq n_{0}(\Gamma, Z)$

$$
\# A_{n}(Z) \sim\left(\frac{1}{\rho^{n}}\right)^{\delta} .
$$

The proof of Lemma 5 follows the lines of Proposition 4 in [S2] and is a consequence of asymptotic estimates of the orbital counting function. For groups of finite co-volume, the case considered in [S2], these estimates are classical [N], for our case (geometrically finite groups) they are due to S.J. Patterson and they are as follows.

Let $\Gamma$ be a discrete group of hyperbolic isometries of the unit ball $B^{n}$. Let $\omega, \eta \in B^{n}$. We define $N(r, \omega, \eta)$ as the number of elements $\gamma \in \Gamma$ such that

$$
\gamma(\eta) \in\left\{x \in \Delta: \rho_{B}(\omega, x) \leq r\right\}
$$

where $\rho_{B}$ denotes the hyperbolic distance in $B^{n}$ associated to the metric $d s=$ $\frac{2|d x|}{1-|x|^{2}}$.

Theorem. If $\Gamma$ is geometrically finite, then there exists $r_{0}>0$ depending on $\omega$ and $\eta$ such that

$$
N(r, \eta, \omega) \sim e^{r \delta} \quad \text { for } r \geq r_{0}
$$

where $\delta$ is the exponent of convergence of $\Gamma$. 


\section{REFERENCES}

[A] L. V. Ahlfors, Möbius transformations, Lecture Notes, Univ. of Minnesota, Minneapolis, 1981.

[B] A. F. Beardon, The geometry of discrete groups, Springer-Verlag, 1983.

[Be] L. Bers, An inequality for Riemann surfaces, Differential Geometry and Complex Analysis H.E. Rauch memorial volume, Springer-Verlag, 1985.

[BJ] C. Bishop and P. Jones, Hausdorff dimension and Kleinian groups, preprint.

[C] L. Carleson, Selected problems on exceptional sets, Van Nostrand, Princeton, NJ, 1967.

[D] S. G. Dani, On badly approximable numbers, Schmidt games and bounded orbits of flows, LMS Lecture Notes, 134, Cambridge Univ. Press, 1989.

[FP] J. L. Fernández and D. Pestana, Radial images by holomorphic mappings, Proc. Amer. Math. Soc. (to appear).

[J] V. Jarník, Zur metrischen theorie der diophantischen approximationen, Prace Mat-Fiz. 36 (1928-1929), 91-106.

[K] I. Kra, Automorphic forms and Kleinian groups, Benjamin, Reading, MA, 1972.

[M] A. Manning, Dynamics of geodesic and horocycle flows on surfaces of constant negative curvature, Ergodic Theory, Symbolic Dynamics and Hyperbolic Spaces, Oxford Univ. Press, 1991.

[N] P. Nicholls, The ergodic theory of discrete groups, LMS Lecture Notes, 143, Cambridge Univ. Press, 1989.

[P1] S. J. Patterson, Diophantine approximation in Fuchsian groups, Philos. Trans. Royal Soc. London 282 (1976).

[P2] - Some examples of Fuchsian groups, Proc. London Math. Soc. 39 (1979), 276-298.

[P3] - The limit set of a Fuchsian group, Acta Math. 136 (1976), 241-373.

[PS] R.S. Phillips and P. Sarnak, On the spectrum of the Hecke groups, Duke Math. J. 52 (1985), 211-221.

[R] C.A. Rogers, Hausdorff measures, Cambridge Univ. Press, 1970.

[Ro] D. Rosen, A class of continued fractions associated with certain properly discontinuous groups, Duke Math. J. 21 (1954), 549-563.

[S1] D. Sullivan, The density at infinity of a discrete group of hyperbolic motions, Publ. Math. Inst. Hautes Etude. Sci. 50 (1979), 171-202.

[S2] D Disjoint spheres, approximation by imaginary quadratic numbers and the logarithm law for geodesics, Acta Math. 149 (1982), 215-237.

[S3] _ Entropy, Hausdorff measures old and new, and limit sets of geometrically finite Kleinian groups, Acta Math. 153 (1984), 259-277.

[T] B. Thurston, Geometry and topology of 3-manifolds, Notes from Princeton University, 1978.

[Tu1] P. Tukia, On the dimension of limit sets of geometrically finite Möbius groups, Ann. Acad. Sci. Fenn. Ser. A I 19 (1994), 11-24.

[Tu2] _ On isomorphisms of geometrically finite Kleinian groups, Publ. Math. Inst. Hautes Ètude Sci. 61 (1985), 171-214.

Departamento de Matemáticas, Universidad Autónoma de Madrid, 28049 Madrid, SPAIN

E-mail address: pando@ccuam3.sdi.uam.es 